\title{
TOWARDS VIRTUAL ASSESSMENT OF HUMAN FACTORS: A CONCEPT FOR DATA DRIVEN PREDICTION AND ANALYSIS OF PHYSICAL USER-PRODUCT INTERACTIONS
}

\author{
Wolf, Alexander; Binder, Nicole; Miehling, Jörg; Wartzack, Sandro \\ Friedrich-Alexander-Universität Erlangen-Nürnberg
}

\begin{abstract}
The early consideration of human factors in product development hugely favours the development of products, which excel with a positive user experience. The virtual environment of product development however, still has significant gaps in the virtual assessment and simulation of human factors, especially for user-product interactions composed of human movements. This motivates us to introduce a concept for data-driven prediction and analysis of user-product interactions. Heart of the concept is a predictive component that models the interaction between the user, represented by a musculoskeletal model, and the product, represented by product characteristics. We describe the implementation of this concept based on a pilot study for a lifting task. Motion capturing was performed to build a database and compare the results of our novel approach. The resulting kinematic and dynamic quantities show similar curve profiles with a small constant offset to the measured data. This indicates that the concept enables the virtual comparison of different designs or concepts regarding human factors.
\end{abstract}

Keywords: User centred design, Simulation, Early design phases, User-product interaction, Data-driven movement prediction

Contact:

Wolf, Alexander

Friedrich-Alexander-Universität Erlangen-Nürnberg

Engineering Design

Germany

a.wolf@mfk.fau.de

Cite this article: Wolf, A., Binder, N., Miehling, J., Wartzack, S. (2019) 'Towards Virtual Assessment of Human Factors: A Concept for Data Driven Prediction and Analysis of Physical User-product Interactions', in Proceedings of the 22nd International Conference on Engineering Design (ICED19), Delft, The Netherlands, 5-8 August 2019. DOI:10.1017/ dsi.2019.410 


\section{MOTIVATION}

The early consideration of human factors in product development hugely favours the development of products, which excel with a positive user experience. As an example, it is important to avoid the occurrence of unexpected discomfort during product use, since this usually results in a negative user experience (Bubb, 2015). In order to ensure a good user experience in current product developments, human factors related requirements (like the absence of discomfort or ergonomics) are usually assessed with user tests. These however, require a physical test environment, prototypes or a predecessor as well as many subjects and are therefore time-consuming and costly. Furthermore, the gathered results are usually subjective. Hence, the results are not holistically back traceable to product characteristics. The main disadvantage of (user-) tests is that the results are created using a physical mock-up (prototype) for whose development certain decisions have already been made. In times of drastically decreasing development times, the tests' results can therefore only have a marginal influence on the final design. This general problem applies to many engineering disciplines and has been known for decades. In order to cope with this problem, digital models are utilised to predict the prospective product behaviour virtually. Accordingly, digital models provide information about the consequences of decisions, regarding product characteristics, in the early phase of product development (Vajna et al., 2018). This approach is already successfully applied in the fields of structural mechanics or dynamics. However, the virtual assessment of a product's design with regard to human factors requires a virtual model of the user-product interaction. The schema shown in Figure 1 generally represents the relationship between a user and a product. The product with its technical, economical and human-related properties interacts with the user, described by demographic and psychographic characteristics, via a process of perception and response (Seeger, 2005). In order to model the user-product interaction holistically, models are necessary that represent the product, the user and the way these models interact. Depending on the assessment's purpose, the models need a different level of detail.

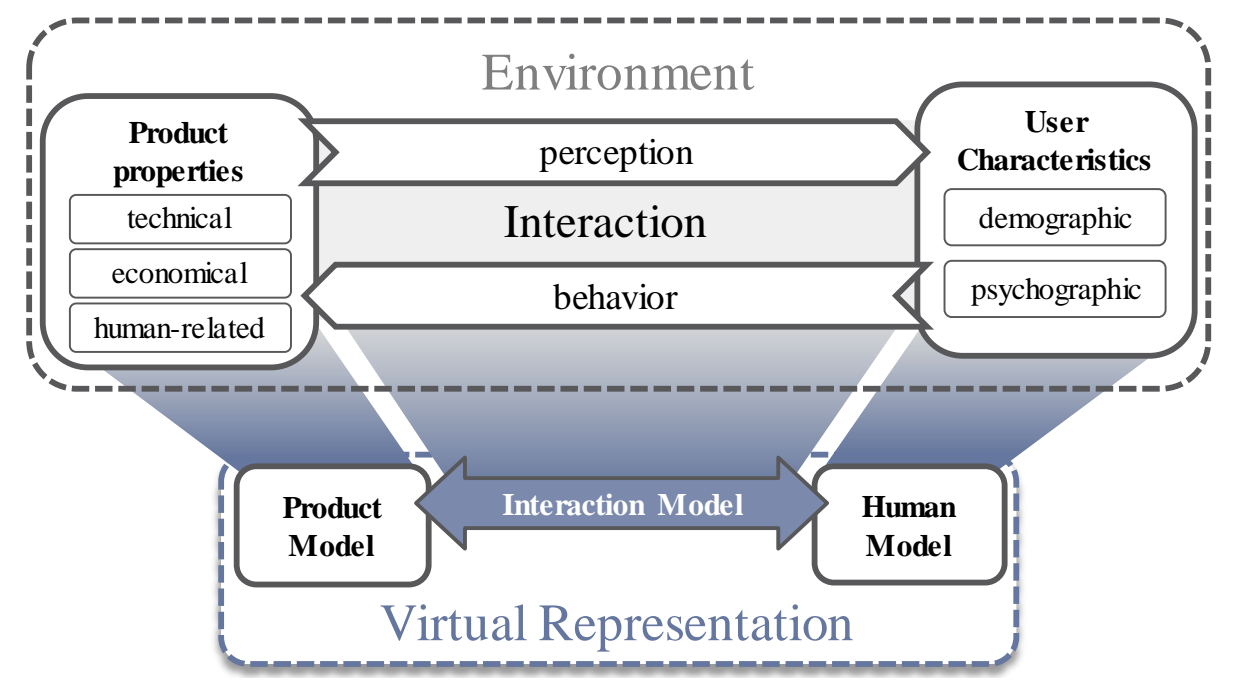

Figure 1: Model of human-product relationships based on (Seeger, 2005) and their virtual
representation

An existing CAE solution for the consideration of human factors are anthropometric human models (e.g. Siemens Jack or Human Builder). These are applicable for the execution of space requirement analyses, movement range analyses or visual analyses. These models are usually part of common CAD-tools. The interaction is modelled by the relative positioning of the human model (in certain postures) to the CAD-model (Miehling et al., 2013). Unfortunately, anthropometric human models lack a sufficient level of detail, concerning the assessment of "dynamic" human factors like discomfort or ergonomics. This especially applies for user-product interactions composed of human movements. According to Zhang et al. (Zhang et al., 2016) discomfort is composed of Fatigue, Restlessness, Pain/ Biomechanics, Strain and Circulation. Although anthropometric digital human models allow for rudimental biomechanical analyses, their applicability is limited to postures, since movements are modelled quasi-static. Thus, they do not consider the velocity, acceleration, and resulting inertia forces 
and moments, which are crucial for biomechanical movement analysis (Wagner et al., 2007). As Rasmussen (Rasmussen, 2005) claims, discomfort is quantifiable through the utilization of musculoskeletal human models. Musculoskeletal human models represent the biomechanical locomotion apparatus using a multi-body dynamics approach. Musculoskeletal simulation tools like the AnyBody Modelling System (Damsgaard et al., 2006) allow the computation of biomechanical quantities (like muscle and joint-reaction forces), which can serve as indicators to assess pain, fatigue or strain dynamically. Musculoskeletal simulations however, rely on experimental data. To analyse a particular movement, motion capture data needs to be acquired in a specially instrumented movement laboratory. This procedure corresponds to the execution of user tests. Accordingly, musculoskeletal simulation currently lack the necessary predictive character to use it as a CAE tool in early product development phases (Miehling et al., 2015). In order to establish musculoskeletal simulation as a CAE-tool nevertheless, a movement prediction component is necessary, which replaces the need for experimental motion capture data.

The understanding and prediction of human movement is subject to many scientific investigations. In character animation, data-driven approaches, using artificial neural networks and reinforcement learning, are showing promising results (Holden et al., 2016; Peng et al., 2018). These methods are applied with the objective of synthesising movements, which appear realistic. They are therefore not inevitably dynamically consistent and physiologically valid, which is necessary for biomechanical analysis. In biomechanics however, numerical approaches using constrained optimization are preferred, since they ensure dynamic consistent and physiological movements, by including the limitations of the human locomotor apparatus via a musculoskeletal model (Farahani et al., 2016; Ackermann and van den Bogert, 2010). These approaches are promising, but they are not yet applicable for product development, since they are too demanding in modelling effort and computation time. Even more problematic is the fact, that these approaches commonly model specific and barely interactive movements such as gait, jumping or running. They are not able to model the choice between different movement strategies. For the purposes of product development, an interaction model is necessary, which predicts physiological movements, depending on user and product characteristics. This motivates us to introduce a concept for prediction and analysis of user-product interactions. Heart of the concept is a predictive component that models the interaction between the user, represented by a musculoskeletal model, and the product, represented by product characteristics. This predictive component combines a data-driven method with a constrained optimization algorithm and shall qualify musculoskeletal simulation as a CAE tool.

\section{METHODS}

\subsection{Overall concept}

The overall concept, depicted in Figure 2, consists of two main parts, the data acquisition part (DAP) and the tool for prediction and analysis of user-product interactions (TPUPI). The DAP has to be executed once in order to build a database, containing different movements of the same interaction. The DAP follows the standard workflow of musculoskeletal simulation: Experimental data (motion capture data) and the musculoskeletal human model serve as input for a kinematic analysis, which computes the movements expressed in the coordinates of the musculoskeletal model (positions/ joint angles). Those kinematic results are further processed and generalized to form the database, which is later used by a regression model, serving as the data-driven part of the predictive component.

The TPUPI provides the possibility for movement prediction and analysis. The TPUPI is thus the actual CAE tool. In principle, the TPUPI follows the same procedure as the DAP, except no experimental data is used as kinematic input. Instead, the predictive component, consisting of two parts, predicts the movement strategy for one particular interaction. The interaction is defined using so-called user-product interaction factors, which also serve as interface for the prospective users of the CAE tool (further explanations in chapter 2.2). The regression model predicts the movement strategy expressed by position-time-curves and/or joint-angle-time curves, based on the movement strategies stored in the database and the user-product interaction factors. The second predictive part is a kinematic movement prediction approach, which transforms these kinematic position-time curves into a whole-body movement expressed in the coordinates of the musculoskeletal model (positions/ joint angles). 


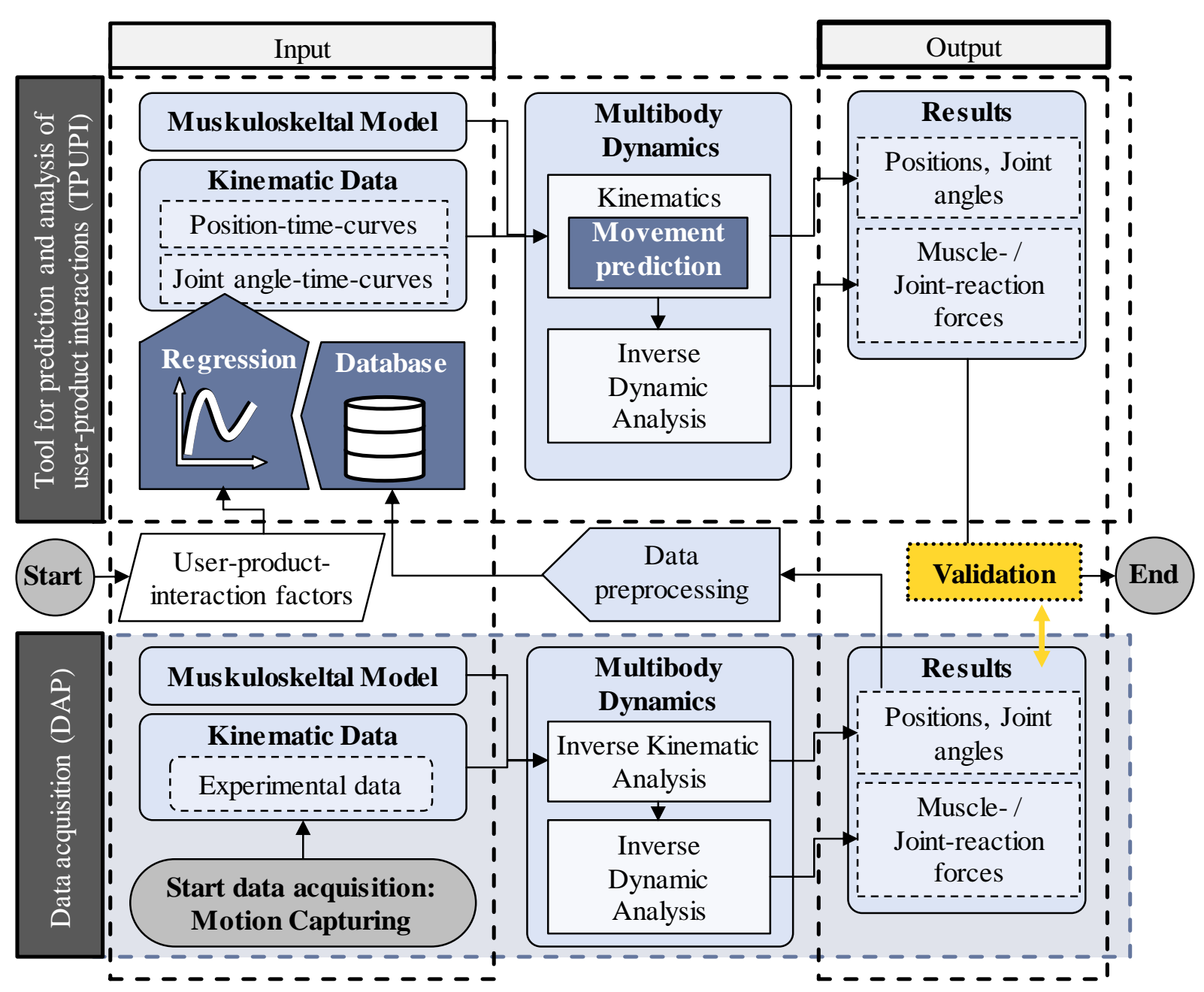

Figure 2. Schematic of the overall concept and approach of this contribution. The dark blue blocks mark the parts of the predictive component. "Start" marks the later usage of the tool, when the database is already build.

The resulting movement can be analysed via an inverse dynamic approach. This approach enables the computation of the desired biomechanical results (muscle and joint-reaction forces). In the same way, the experimental movement data used in the DAP can be dynamically analysed. This provides the opportunity to compare the results of the standard workflow of musculoskeletal simulation with our new approach (CAE-tool). As a pilot study, this concept was implemented for a symmetrical lifting task (lifting of an object in the sagittal plane).

\subsection{Data collection using motion capture}

In order to model the choice between different movement strategies with data-driven approaches, training data is required, which contains various movements for different scenarios of the same interaction. For the pilot study, the predictive component of the approach shall "learn" the movement strategies of human symmetrical lifting. For the pilot study, it is assumed that the choice of a movement strategy is influenced by the following user-product interaction factors:

- The anthropometry of the user

- The movement task, which needs to be fulfilled

- The stress, the user is exposed to during interaction

Based on these user-product interaction factors, a design of experiments (DoE) was set up for a motion capturing study of different lifting movements. To represent the lifting movements quantitatively, the body height of the subjects abstracted the anthropometry, spatial specifications (depicted in Figure 3) abstracted the movement task and the weight of the lifting object abstracted the stress. Two healthy subjects ( 1 female/ 1 male, age $24 / 25$ years, body height $1.74 \mathrm{~m} / 1.96 \mathrm{~m}$ ) volunteered for the motion capture study. Since it is the concept of DoE to describe an entire system behaviour with as little experimental data as possible, three completely different movement tasks were chosen to cover the 
possible design space of a symmetric lift in the best possible way (Figure 3). The object weight was varied in three steps: $2 \mathrm{~kg}, 10 \mathrm{~kg}$, and $20 \mathrm{~kg}$. Using a full factorial design $(3 \times 3 \times 2$ levels), 18 unique lifting movements were captured. The motion capturing was performed, using a Qualisys motion capture system. 34 reflective markers were attached to the subjects. Of these, eight markers were attached to each upper extremity, five to each lower extremity, and four to the front and rear of the upper body. Nine infrared high-speed cameras (sampling rate $60 \mathrm{~Hz}$ ) recorded the marker trajectories. The kinematic data was stored in 18 C3D-files using the Qualisys Track Manager 2.11.
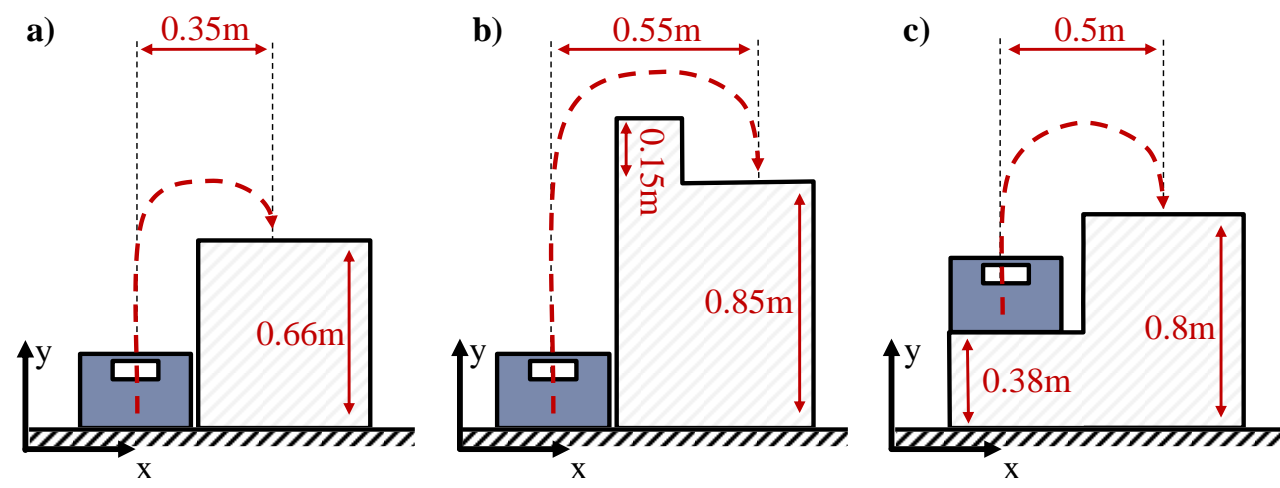

Figure 3. Three different lifting tasks, abstracted with quantitative spatial specifications a) standard lift; b) edge lift; c) shortened lift

The test persons were instructed to perform the lift with a straight back from the legs without taking a step forward. In addition, they were instructed to adhere to these guidelines only as long as they felt safe with it, in order not to endanger their health. In the end, five movements could not be performed in accordance with these guidelines. Subject 2 was not able to perform the edge lift with $20 \mathrm{~kg}$ without an additional step forward. Likewise, subject 1 had to perform a step forward to execute the edge lift with 2,10 and $20 \mathrm{~kg}$ as well as the standard lift with $20 \mathrm{~kg}$.

\subsection{Data acquisition part}

The trajectory data stored in the $18 \mathrm{C} 3 \mathrm{D}$ files was further processed using the AnyBody Modelling System (Damsgaard et al., 2006). The GaitFullBody-Model from the AnyBody Managed Model Repository (AMMR) 1.6.3. was utilized to animate the musculoskeletal model using the marker trajectories. In a first step, the musculoskeletal model was scaled to the anthropometries of the subjects using the parameter identification method (Andersen et al., 2010). In a second step, the experimental movement data was fitted upon the scaled models using an inverse kinematic analysis. Output of this analysis is the full-body movement expressed in the coordinates of the musculoskeletal model (time series of joint angles and time series of the relative position of the pelvis to a global coordinate system).

Additionally, time series of the $\mathrm{x}$ - and y-position of the centre of mass (CoM) and of the hands over time were computed via the kinematic analyses. Those data series, together with the joint angle values of the knee and elbow flexion, were extracted from the kinematical results. The extracted data (18x6 data series with different durations) has been normalized in time. In addition, the values of the position data series were generalized. The data series containing movements with a "step forward" were sorted out, since they would bias the database and therefore the regression model. The joint angle data was stored in the database directly. In contrast, the $\mathrm{x}$ - and $\mathrm{y}$-position data of the CoM and the Hands were stored indirectly as a degree of fulfilment (DF) according to Formula 1.

$$
D F_{i}=\frac{D P_{i}}{T V}
$$

The degree of fulfilment defines which proportion of the target value (TV) the data point (DP) has reached at a certain time step $\mathbf{i}$. The target value of the data series of the box in y-direction (height) is for example the final height of the object (e.g. $0.66 \mathrm{~m}$ for the standard lift scenario). This relative consideration was introduced to increase the stability of the kinematic movement prediction approach (of the TPUPI) since this approach uses the target values in its objective function. Processed in this way, the data of the remaining movements were stored in the database (13x6 data series). Additionally to every movement, the abstracted user-product interaction-factors as well as the movements' durations were stored, in order to assign the kinematic data to their associated interactions. 


\subsection{Tool for prediction and analysis of user-product interactions}

The user-product interaction factors introduced in chapter 2.2, also serve as an abstract virtual product representation:

- The anthropometry describes the product's target group

- The movement task describes the product's kinematics

- The stress contains the forces and moments a user must exert in order to use a product

With the same kind of abstraction as with the DoE, these factors enable the product designer to rudimentarily describe relevant product characteristics with a user-product interaction. Via the assignment of concrete (scalar) values to these factors, a particular interaction is specified, for which the predictive component of the TPUPI is supposed to predict a unique movement. The dynamic results of the corresponding movement analysis are therefore holistically back traceable to the product's characteristics. This way, different product designs and concepts become comparable with respect to discomfort and other human factors, since the user-product interaction factors are coupled to the product characteristics.

\subsubsection{Regression model}

The regression model is the first part of the predictive component. Purpose of the regression model is predicting unique and physiologically reliable kinematic data (movement strategy) for any set of feasible user-product interaction-factors. In the pilot study, a Support Vector Machine (SVM), a supervised machine learning tool used in classification and regression (Vapnik, 2000), was used as regression model. For training, the user-product interaction factors, describing the interactions of the experimental data, served as predictors, while the experimental movement data served as target values. Once trained, the SVM is able to predict movement strategies (position-time-curves/ joint-angle-time curves) for arbitrary feasible user-product interaction-factors (which define new unknown interactions). Since the movement data in the data base is normalized regarding time, the regression model additionally predicts the movements' durations.

For a first evaluation of the predictive component, the SVM was trained with the database using a polynomial kernel function and an automatic kernel scale. The trained SVM was utilized to reconstruct the captured movements, which are described in chapter 2.2. To do so, user-product interaction factors representing the captured movements were defined as predictors, to compute new elbow/ knee flexion angles and $\mathrm{x}$ - and y-positions for the hands and the CoM (18x6 data series).

\subsubsection{Movement prediction approach}

The movement prediction approach is the second part of the predictive component, with the purpose of computing a whole-body movement from the few kinematic data series provided by the regression model (which contain the movement strategy).

The movement prediction approach is based on a method presented in one of our previous contributions (Wolf and Wartzack, 2018). The approach itself depends on the hypothesis that not the complete kinematic data, describing the degrees of freedom of the human model, has to be present a priori in order to produce a physiologically plausible and physically consistent movement. Rather, it may be sufficient to kinematically drive certain "leading parts" of the human body (Bubb et al., 2006). The regression model predicts the data that drives these leading body parts. These are defined as major constraints in an over-determinate kinematics analysis (Andersen et al., 2009), which is implemented in the AnyBody Modelling System. The joint angles of the human body contain dummy values, which are defined as minor constraints in the over-determinate kinematic analysis. This method allows the computation of the best possible fit between kinematic constraints in a kinematically over-determinate system (more kinematic constraints than degrees of freedom). It additionally allows the definition of a weight function for each constraint, what enables the definition of major and minor constraints.

In the pilot study, the hands, the feet and the CoM, as well as the elbow and knee flexion, were considered as the "leading body parts" of the symmetric lifting movement. The feet were constrained to stay on the ground. The time dependent positions of the hands and the CoM, as well as the time dependent joint angles of the elbow and the knee were constrained to the data series predicted from the regression model. The undefined joint angles remaining, adjust to the principle of skeletal support. This principle rests upon the assumption, that the human body chooses movement strategies in such a way, the skeletal system maximally absorbs the external strain. Accordingly, the approach results in 
movement strategies trying to flex the joints as little as possible from the "neutral position". When all joints are in the "neutral position", the musculoskeletal model takes an upright posture with hanging arms. This principle was implemented by constraining each dummy value of the joint angles to the neutral position. These constraints were defined with a minor weight, so they can be violated in order to obey the major constraints of the leading body parts. Additionally, these minor constraints were weighted differently to each other. "Strong" joints (joints actuated by strong muscles) were weighted weaker than "weaker" joints (joints actuated by weaker muscles respectively). This ensures that a "strong" joint flexes more likely than a "weak" joint. With this movement prediction approach, 18 lifting movements (full body kinematics described in joint angles/ pelvis position) were predicted based on the data series computed by the regression model.

\subsubsection{Dynamic analysis}

Finally, an inverse dynamic analysis was performed to compute dynamic biomechanical quantities like muscle- and joint reaction forces. Additional to the kinematics (movement), the dynamic interaction with the environment (external forces and moments) is a necessary input for the inverse dynamic analysis (Damsgaard et al., 2006). For the pilot study, a predictive contact model (Fluit et al., 2014) was used to compute the ground reaction forces. Default reaction forces modelled the weight of the object acting on the hands. The muscles were modelled using the AnyMuscleModel. In order to identify the muscular forces, the default muscle recruitment solver of the AnyBody Modelling System (Damsgaard et al., 2006) was used. The inverse dynamic analysis was performed for all the captured lifting movements (DAP) and all the predicted lifting movements (TPUPI).

\section{RESULTS}

\subsection{Kinematic results}

Figure 4 compares selected kinematic results of the data acquisition part (based on the captured data) with the kinematic results of the tool for prediction and analysis of user-product interactions.

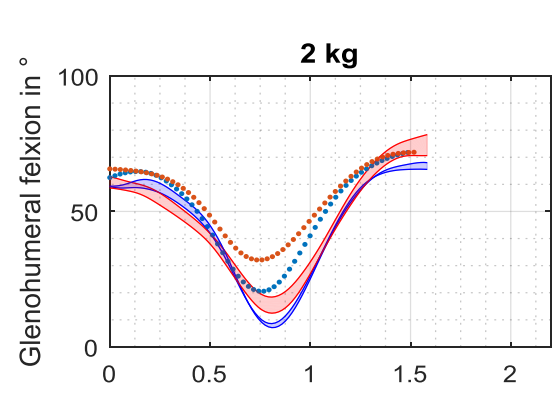

a)

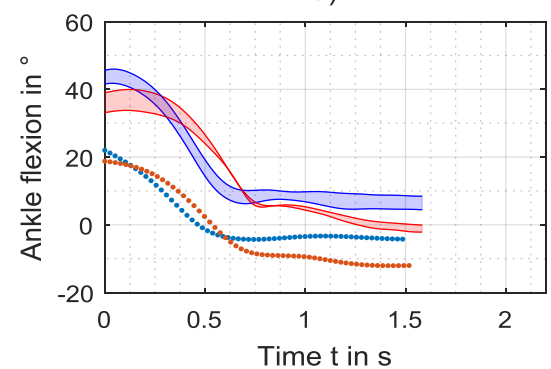

d)

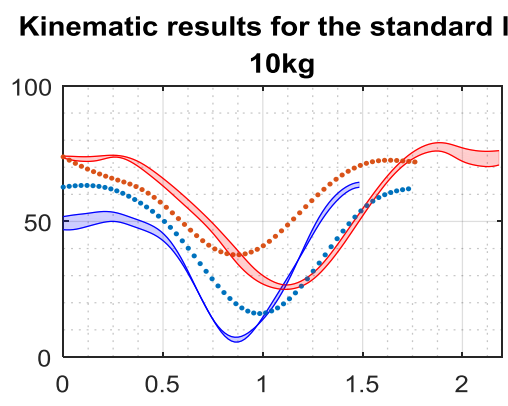

b)

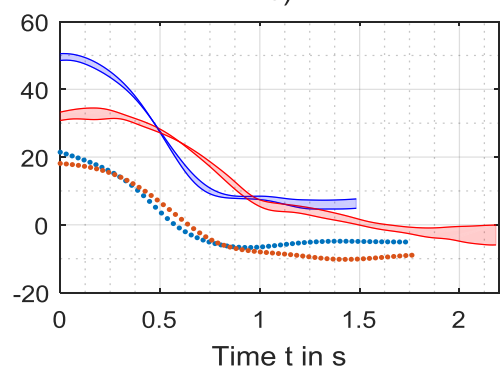

f)

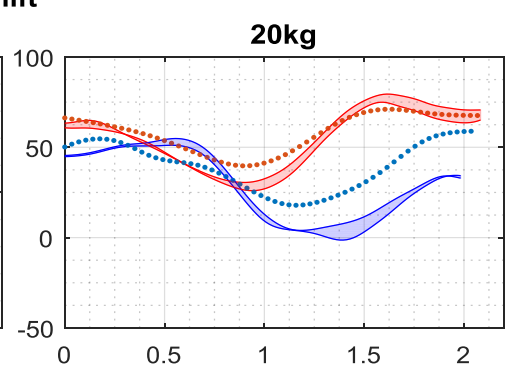

c)

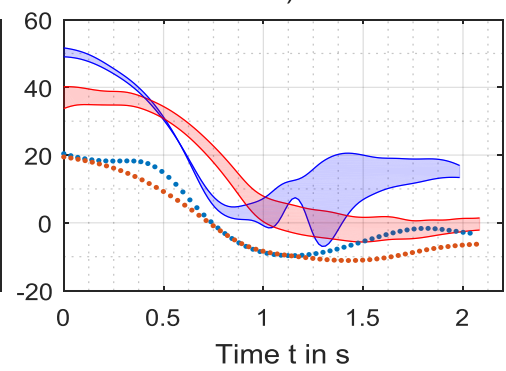

g)

DAP subject $1 \square$ DAP subject $2 \cdot$ TPUPI subject $1 \bullet$ TPUPI subject 2

Figure 4. Comparison of the computed ankle- and glenohumeral flexion angles from the DAP and the TPUPI for both subjects and different weights. The shaded area represents the range between the left and the right extremities' joint angles of the experimental data.

The figure shows that the TPUPI results in similar joint angle curve profiles to the DAP, which though differ in magnitude. All the predicted joint angles have a certain and mostly constant offset to the measured data. Exceptions are the elbow and the knee flexion. They have nearly the same curve 
profile and the same magnitude, since these, as well as the positions of the hands, feet and CoM are constrained directly to the data predicted by the regression model. This shows that the regression model was able to reproduce the cases it was trained with. It additionally leads to the conclusion that the principle of skeletal support "chooses" a different kinematic chain (which describes the joint angles from the feet to the hands) from the range of possibilities to fulfil the movement task (Figure 5). As observable from Figure $4 \mathrm{~g}$ ), subject 1 did a step forward during the motion capturing of this particular movement. Although this data was excluded when training the regression model, Figure 4 c)/g) shows that the predicted curve profile of subject1 differs insignificantly from the predicted curve profile of subject 2. The comparison with the curves in Figure 4 a)/d) and b)/ c) indicates that the regression model works well for new "unknown" cases. The predicted interaction durations in Figure $4 \mathrm{~b}) / \mathrm{c})$, however, indicate that the regression model needs more data to predict the interactions duration more accurately.

\subsection{Dynamic results}

Figure 5 shows the dynamically analysed movement of the TPUPI and the DAP and compares a predicted movement with the measured one at certain percentages of the movement's duration.

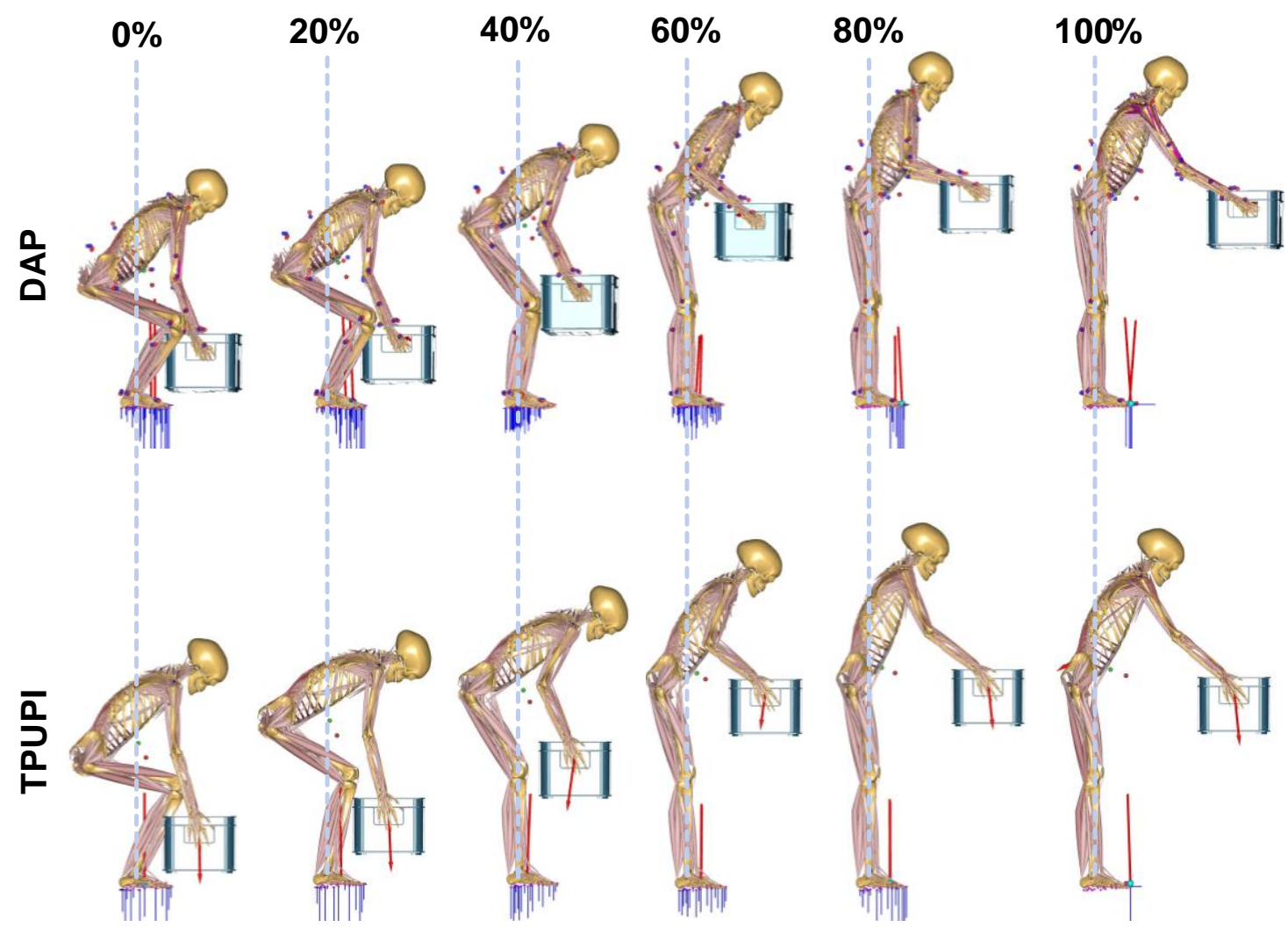

Figure 5. Results of the dynamic analysis using experimental data (top) and the introduced movement prediction approach (bottom) for the standard lift of subject 1 with $10 \mathrm{~kg}$

Figure 6 compares the computed intradiscal compression force between the fourth and the fifth lumbar vertebrae (an indicator for discomfort in the spine) and the computed residual forces. For better comparability, the graph shows the time integral (area beneath the curve) of the L4-L5 compression force and the time integral of the residual force vector. The residual forces are responsible for balancing any dynamic inconsistencies. If the human model drops out of dynamic equilibrium (balance), due to measurement errors or movement fitting losses, the residual forces intervene to maintain the dynamic equilibrium. These are therefore an indicator of whether a movement prediction was successful. Since the residual forces support and relieve the body during movement, the dynamic results are biased to certain extent (depending on the residual forces' magnitude) in the time intervals they act. During the lifting movements, the residual forces occur only in the time interval during the forward bending (to put the box down). The forces are correspondingly high when interacting with the $20 \mathrm{~kg}$ object. This applies for the DAP results as well as for the TPUPI results. While the residual 
forces are low (as they are during the lifting interval), the calculated L4/L5 compression forces of the TPUPI show a constant offset (with higher forces) to the results of the DAP.

The "step forward"-movement highlighted in Figure 6 shows a new predicted movement, since the corresponding data was sorted out for the regression model training. The residual forces of this movement are very high. This shows that this particular interaction is actually out of the design space, covered by the current abstraction of the movement task (chosen user-product interaction factors). In order to predict this interaction, the musculoskeletal model, should be allowed to take a step forward in order not to lose its balance. To extend the model in this sense, the step forward would have to be considered in the abstraction of the movement task. In addition, the regression model would have to be additionally trained with position data of the foot, which also would have to be considered as leading body part in the movement prediction component.

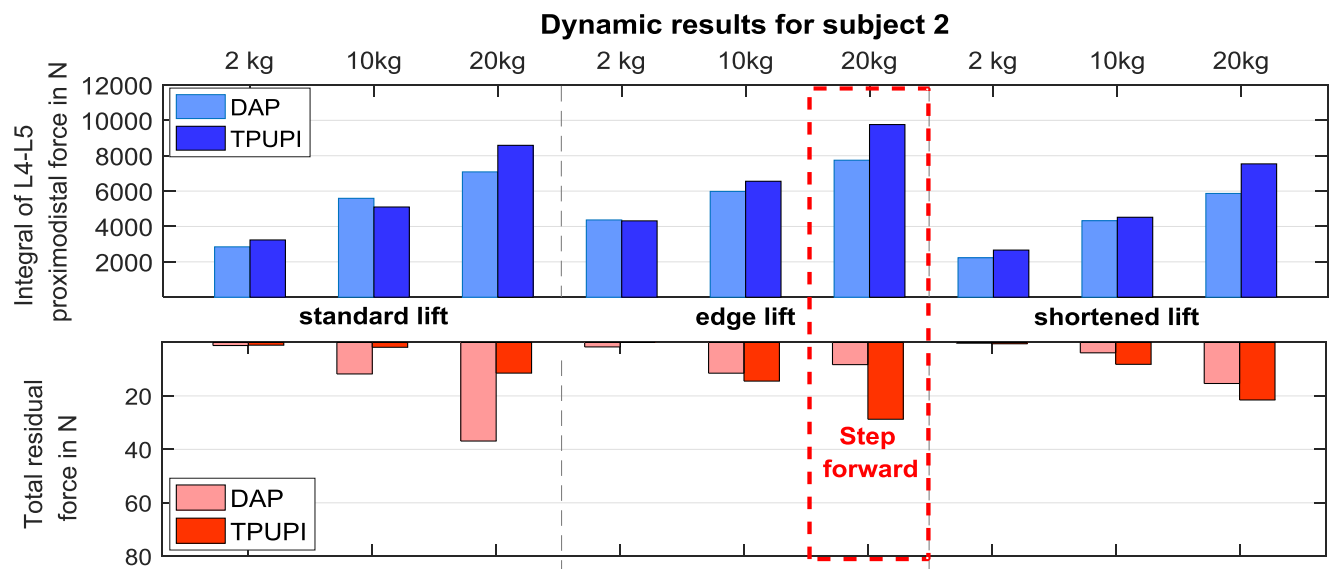

Figure 6. Comparison of the L4-L5 compression forces and residual forces

\section{DISCUSSION}

The pilot study provides initial indications whether the presented approach is valid. The suitability of the predictive components (SVM as regression model and the movement prediction approach) need to be evaluated separately, using sensitivity and validation studies. In addition, it must be determined whether more "leading body parts/ joint angles" in the movement prediction part guarantee a more stable/ better prediction. The overall concept needs to be validated using different training and test datasets. Motion capturing and musculoskeletal simulations are demanding and time consuming. To demonstrate feasibility within the presented pilot study, all available data were used to train the regression model in order to achieve the best possible prediction outcome.

Nevertheless, the results of the pilot study show that the overall concept produces first reasonable results. The kinematic and thus the dynamic results show a constant offset to the results computed via the standard workflow of musculoskeletal simulation. The predictive component generates physiological reasonable movements with mostly low residual forces, but is not yet able to find the most efficient movement possibility. While the regression model was able to reproduce the interaction behaviour well, the principle of skeletal support does not seem to model real movement strategies satisfactorily. The specification of a fixed value (neutral position) as a target value seems to generate the offset between the generated joint angles and the measured ones. The offset in the dynamic results is a consequence of this kinematic offset. Further research is required to explore appropriate objective functions. The approach with the strengthweighted joints proves to be promising, since the predicted joint angle curve profiles show clear similarities with the measured ones. Although the absolute result values of the TPUPI and the DAP differ in comparison, the relative differences between the individual interactions are similar. Therefore, the tool seems to compare different designs and their matching interactions reliably.

\section{SUMMARY AND OUTLOOK}

The overall concept may enable product developers to virtual assess discomfort and other human factors quantitative and holistically back traceable. Since the modern product development is characterized by the reuse and adaptation of existing solutions/designs (Gunduz and Yetisir, 2018), a one-time training and modelling effort seems worthwhile to avoid users test, with all its drawbacks. 
The presented CAE tool can enrich the virtual environment of product development by offering the possibility to address human factors in early product development phases.

\section{REFERENCES}

Ackermann, M. and van den Bogert, A.J. (2010), "Optimality principles for model-based prediction of human gait", Journal of biomechanics, Vol. 43 No. 6, pp. 1055-1060.

Andersen, M.S., Damsgaard, M., MacWilliams, B. and Rasmussen, J. (2010), “A computationally efficient optimisation-based method for parameter identification of kinematically determinate and over-determinate biomechanical systems", Computer methods in biomechanics and biomedical engineering, Vol. 13 No. 2, pp. 171-183.

Andersen, M.S., Damsgaard, M. and Rasmussen, J. (2009), "Kinematic analysis of over-determinate biomechanical systems", Computer methods in biomechanics and biomedical engineering, Vol. 12 No. 4 , pp. 371-384.

Bubb, H. (2015), Automobilergonomie, ATZ/MTZ-Fachbuch, Springer Vieweg, Wiesbaden.

Bubb, H., Engstler, F., Fritzsche, F., Mergl, C., Sabbah, O., Schaefer, P. and Zacher, I. (2006), “The development of RAMSIS in past and future as an example for the cooperation between industry and university", International Journal of Human Factors Modelling and Simulation, Vol. 1 No. 1, pp. 140.

Damsgaard, M., Rasmussen, J., Christensen, S.T., Surma, E. and Zee, M.d. (2006), "Analysis of musculoskeletal systems in the AnyBody Modeling System", Simulation Modelling Practice and Theory, Vol. 14 No. 8, pp. 1100-1111.

Farahani, S.D., Andersen, M.S., de Zee, M. and Rasmussen, J. (2016), "Optimization-based dynamic prediction of kinematic and kinetic patterns for a human vertical jump from a squatting position", Multibody System Dynamics, Vol. 36 No. 1, pp. 37-65.

Fluit, R., Andersen, M.S., Kolk, S., Verdonschot, N. and Koopman, H.F.J.M. (2014), "Prediction of ground reaction forces and moments during various activities of daily living", Journal of biomechanics, Vol. 47 No. 10, pp. 2321-2329.

Gunduz, M. and Yetisir, T. (2018), "A design reuse technology to increase productivity through automated corporate memory system", Neural Computing and Applications, Vol. 29 No. 9, pp. 609-617.

Holden, D., Saito, J. and Komura, T. (2016), "A deep learning framework for character motion synthesis and editing", ACM Transactions on Graphics, Vol. 35 No. 4, pp. 1-11.

Miehling, J., Krüger, D. and Wartzack, S. (2013), "Simulation in Human-Centered Design - Past, Present and Tomorrow", In: M. Abramovici and R. Stark, (Ed.), Smart Product Engineering, Lecture Notes in Production Engineering, Springer Berlin Heidelberg, Berlin, Heidelberg, pp. 643-652.

Miehling, J., Schuhhardt, J., Paulus-Rohmer, F. and Wartzack, S. (2015), "Computer Aided Ergonomics Through Parametric Biomechanical Simulation”, Proceedings of the ASME International Design Engineering Technical Conferences and Computers and Information in Engineering Conference - 2015, Boston, Massachusetts, USA, Sunday 2 August 2015.

Peng, X.B., Abbeel, P., Levine, S. and van de Panne, M. (2018), "DeepMimic. Example-Guided Deep Reinforcement Learning of Physics-Based Character Skills", ACM Transactions on Graphics, Vol. 37 No. 4, pp. 1-14.

Rasmussen, J. (2005), "Musculoskeletal Simulation - (Dis)comfort Evaluation", S\&V OBSERVER, pp. 8-9.

Seeger, H. (2005), Design technischer Produkte, Produktprogramme und -systeme: Industrial Design Engineering, 2., bearb. und erw. Aufl., Springer-Verlag Berlin Heidelberg, Berlin, Heidelberg.

Vajna, S., Weber, C., Zeman, K., Hehenberger, P., Gerhard, D. and Wartzack, S. (2018), CAx für Ingenieure: Eine praxisbezogene Einführung, SpringerLink Bücher, 3., vollständig neu bearbeitete Auflage, Springer Vieweg, Berlin, Germany.

Vapnik, V.N. (2000), The Nature of Statistical Learning Theory, Statistics for Engineering and Information Science, Second Edition, Springer, New York, NY.

Wagner, D.W., Reed, M.P. and Rasmussen, J. (2007), "Assessing the Importance of Motion Dynamics for Ergonomic Analysis of Manual Materials Handling Tasks using the AnyBody Modeling System", SAE Congress: Digital Human Modeling for Design and Engineering (DHM).

Wolf, A. and Wartzack, S. (2018), "Parametric movement synthesis. Towards virtual design optimistaion of man-machine interaction in engineering design", Design 2018: Proceedings of the 15th International Design Conference, May 2018, Dubrovnik, Croatia, May, 21-24, 2018, pp. 941-952.

Zhang, L., Helander, M.G. and Drury, C.G. (2016), "Identifying Factors of Comfort and Discomfort in Sitting", Human Factors: The Journal of the Human Factors and Ergonomics Society, Vol. 38 No. 3, pp. 377-389.

\section{ACKNOWLEDGMENTS}

The authors gratefully acknowledge the financial support of project WA 2913/31-1 by the German Research Foundation (DFG). 